\title{
Quelques considérations sur l'évaluation technico-économique des choix de protection radiologique
}

\author{
A. OUDIZ, J. LOMBARD* \\ (Manuscrit reçu le 16 avril 1984)
}

RÉSUMÉ

\begin{abstract}
Cet article propose une interprétation du principe d'optimisation dans une perspective pragmatique qui est celle de l'évaluation technicoéconomique des choix de protection radiologique. Ce processus comporte plusieurs étapes qui sont présentées brièvement et dont on discute ensuite l'importance relative, compte tenu de l'impact économique des choix de protection que l'on soumet à l'évaluation.
\end{abstract}

\section{ABSTRACT}

This article suggests the interpretation of the so-called optimization principle within the pragmatic point of view of a technical and economical assessment of the radiological protection options. This process includes several steps which are briefly presented. Their relative importance is then discussed, taking into account the economical impact of the alternative options.

\section{INTRODUCTION}

Le principe général de la procédure dite d'optimisation est désormais bien connu des spécialistes de la protection radiologique [2]. Bien connue en tant que principe, il n'en reste pas moins que l'optimisation suscite aujourd'hui encore bien des réserves et du scepticisme quant aux perspectives qu'elle offre au plan opérationnel. On se propose dans ce qui suit d'apporter un éclairage concret du contenu de ce principe, susceptible de faire apparaître plus clairement qu'il s'agit d'un concept d'action et non d'un concept théorique sans portée pratique. Au niveau de la terminologie, il convient de garder à l'esprit l'identité du principe d'optimisation et de l'objectif : "maintenir les expositions aussi bas que possible compte tenu des contraintes économiques et sociales". Cette formulation développée du principe d'optimisation souligne d'ailleurs mieux sa portée essentiellement pragmatique.

* Centre d'étude sur l'évaluation de la protection dans le domaine nucléaire (CEPN), BP 48, 92260 Fontenay-aux-Roses. 
Jusqu'à présent, la présentation générale de l'optimisation a été faite de telle sorte qu'elle attache de manière apparemment indissociable ce principe à deux notions "non classiques" en matière de radioprotection, à savoir l'équivalent de dose collectif et l'analyse coût-avantage. Or, cet amalgame est largement infondé, comme l'attestent clairement les récents développements de la doctrine présentés par la CIPR dans sa publication 37 [3] lesquels montrent qu'il conviendra, à l'avenir, de prendre ses distances par rapport à une définition de l'optimisation fondée uniquement sur l'analyse coût-bénéfice et sur l'équivalent de dose collectif.

Ces deux notions ne sauraient épuiser à elles seules le contenu du principe d'optimisation, plus général en réalité et aussi plus pragmatique que la présentation qui en est faite habituellement ne le suggère. Une formulation plus appropriée du principe serait à cet égard souhaitable ; on propose de remplacer le terme "optimisation" par "évaluation technicoéconomique des choix de protection radiologique".

\section{UNE PRÉSENTATION PLUS PRAGMATIQUE DU PRINCIPE D'OPTIMISATION}

Le principe gagnerait sans doute en crédibilité si, pour l'exposer, on mettait l'accent sur le processus qu'il met en jeu, plutôt que sur certaines de ses composantes techniques, telles que l'indicateur de risque ou la méthode permettant le choix des options.

Le principe d'optimisation peut être assimilé, en effet, à un processus comportant plusieurs étapes, comme le montre la figure 1.

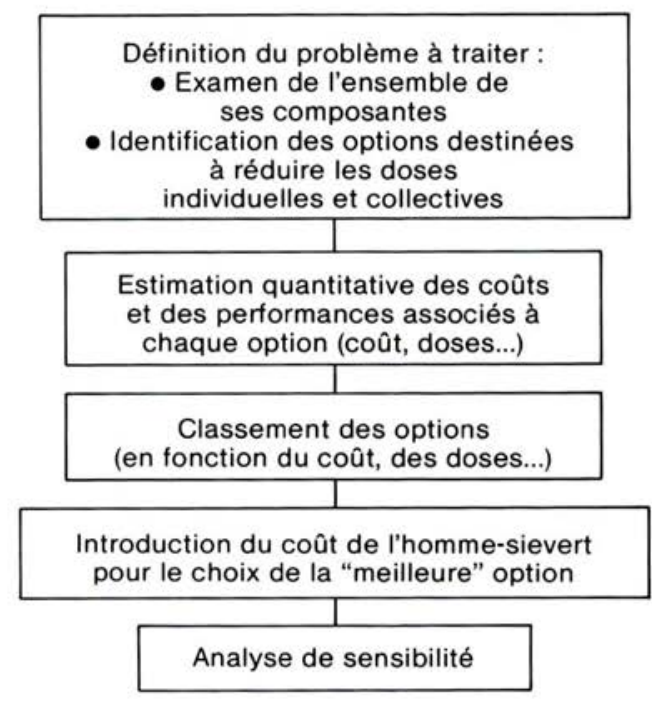

Fig. 1. - L'optimisation en tant que processus séquentiel. 


\section{I.1. La définition du problème à traiter}

La première étape a pour objet la définition précise du problème de radioprotection à traiter. Elle permet, notamment, de passer en revue l'ensemble des dimensions du risque radiologique pertinentes. La dose collective n'est pas nécessairement le seul indicateur du risque dont il faut tenir compte ; ainsi, par exemple, la distribution des doses individuelles pourra jouer un rôle important dans le cas de la protection des travailleurs [9], de même, la distribution temporelle des doses [10] ainsi que la probabilité de survenance d'une dose individuelle maximale constitueront les éléments clés d'une étude d'évaluation technico-économique relative, par exemple, à un stockage de déchets de longue période.

Par ailleurs, parmi les dimensions du risque à prendre en compte, il y aura lieu d'intégrer, le cas échéant, le risque induit par les actions de protection : on songe ici, par exemple, au transfert de risque du public vers les travailleurs consécutif à la mise en œuvre des systèmes de traitements des effluents dans les installations nucléaires [11].

Outre l'examen de l'ensemble des composantes du problème, cette première étape a trait à l'identification des options destinées à réduire le risque radiologique dans ses diverses composantes. II convient à ce stade de distinguer deux modalités de réduction des doses selon que l'action porte sur le débit de dose et/ou la durée d'exposition.

Les actions évoquées le plus fréquemment influent sur les débits de dose : - par réduction de l'activité des sources : ainsi, par exemple, les actions touchant à la composition des alliages métalliques des circuits ou les systèmes de purification de l'eau primaire dans les réacteurs permettent de réduire l'exposition professionnelle; de même, la décontamination des effluents ou le renforcement du confinement des déchets réduisent l'exposition du public ;

par renforcement des protections biologiques : écrans en béton, en plomb, ventilation, etc.

Mais d'autres actions existent aussi, qui n'ont pas, jusqu'à présent, fait l'objet de la même attention que les précédentes, ce sont celles qui influent sur la durée de l'exposition professionnelle individuelle et/ou collective. On pense ici aux actions qui concernent l'automatisation de certaines opérations, l'entraînement du personnel avant certaines interventions particulièrement irradiantes et plus généralement à l'organisation du travail [8].

\section{I.2. Quantification des performances et des coûts}

Cette deuxième étape a pour objet :

1. L'estimation quantitative des coûts d'investissement, d'exploitation-maintenance associés aux diverses options, en tenant compte bien entendu des gains éventuellement obtenus sur le plan de la production (l'automatisation de certaines opérations, l'entraînement du personnel contribuent à raccourcir le temps d'immobilisation de l'unité de production) [8]. 
2. L'estimation quantitative des performances des options de protection, qu'il s'agisse de la réduction des doses collectives et individuelles, mais aussi, si nécessaire, de la modification de la distribution des doses, de la probabilité de leur survenance (cas des déchets notamment) et, le cas échéant, des accroissements de dose entraînés par l'option de protection (cas où il existe un transfert de risque) [11].

\section{I.3. Le classement des options}

La troisième étape concerne le classement des options en fonction de leur coût et de leurs performances.

Tout d'abord, il est utile de noter que certaines options peuvent être parfois éliminées d'emblée, soit parce que les doses individuelles qui subsisteraient après leur mise en œuvre ne permettent pas de satisfaire le $3^{\text {e }}$ principe dit de limitation de dose (cas des travailleurs ou encore du public, en relation avec les déchets de longue période), soit parce que d'autres options de même coût présentent de meilleures performances, soit encore parce qu'elles présentent d'autres inconvénients notables dans d'autres domaines (sûreté, fiabilité, facilité d'entretien...).

La procédure de classement sera choisie en fonction de la multidimensionnalité du problème : si la performance des options est décrite de façon satisfaisante à l'aide de l'indicateur de dose collective, alors le recours à une analyse simple qui compare l'augmentation du coût de la protection à la réduction de l'exposition collective associée aux diverses actions envisageables est largement suffisante. L'analyse coût-efficacité est, dans la plupart des cas, l'outil appoprié pour effectuer le classement de telles options [12].

$\mathrm{Si}$, par contre, il est souhaitable de prendre en considération plusieurs dimensions très hétérogènes, comme cela peut être le cas à propos des déchets de longue période, il est alors préférable de recourir à des méthodes d'aide à la décision plus performantes qui permettent l'identification des actions les plus intéressantes du point de vue de l'ensemble des critères retenus. Les diverses méthodes d'analyse multicritère sont, dans ce cas, les mieux adaptées [13].

\section{I.4. Choix de la "meilleure" option}

Le choix d'une option parmi l'ensemble des alternatives ne constitue en définitive que l'étape ultime du processus d'évaluation technico-économique de la radioprotection. Ce choix implique en général, et on verra plus loin que ce n'est pas toujours indispensable, la référence au coût "alpha" de l'homme-sievert. On ne tentera pas ici de fournir une réponse en termes d'estimation monétaire de "alpha". De nombreux travaux ont été consacrés par ailleurs à cette question, qui mérite des développements spécifiques [6, 7, 10], et ceci d'autant plus que la multidimensionnalité des problèmes de radioprotection rend nécessaire la connaissance de "alpha" mais aussi d'autres termes dits "en bêta", conformément aux développements récents de la doctrine de la CIPR $[1,5,9,10]$. Ces différents 
termes "bêta" permettent d'élargir la méthode coût-bénéfice afin de prendre en considération la distribution des doses individuelles ou la répartition temporelle des risques.

\section{I.5. L'analyse de sensibilité}

Les étapes précédentes nécessitent de nombreuses hypothèses (choix des actions, de leurs performances, de la valeur de l'alpha...) qui influent grandement sur le choix de la "meilleure" option. Le recours à une analyse de sensibilité, qui teste la variabilité du résultat vis-à-vis de diverses modifications de ces hypothèses, peut être nécessaire afin d'avoir une meilleure appréciation de la stabilité du résultat fourni par la méthode d'aide à la décision. Cette étape de vérification pourra également fournir des renseignements précieux quant aux hypothèses ayant le plus de poids sur le résultat final.

\section{NIVEAUX DÉCISIONNELS ET RÉFÉRENCE A LA VALEUR DE "ALPHA”}

On fera ici quelques observations quant à la signification de la valeur de l'homme-sievert et à l'utilisation de ce concept, selon l'importance économique relative des diverses décisions de radioprotection.

\section{II.1. Signification du coût de l'homme-sievert alpha}

Rappelons tout d'abord que le coût de l'homme-sievert alpha est l'équivalent monétaire associé à l'unité de dose collective.

Le coût de l'homme-sievert alpha est une valeur dé référence à laquelle on compare le rapport coût-efficacité d'une option "marginale". En théorie, l'option sera retenue si le rapport coût-efficacité est inférieur ou égal à alpha et rejetée dans le cas contraire. L'exemple présenté dans l'encadré ci-après permettra d'illustrer la notion d'option marginale qui est ùne condition, souvent mal connue, de l'application de ce concept en vue de procéder au choix.

\section{Exemple}

Supposons que le problème d'évaluation consiste à choisir le nombre de filtres, tous identiques (de coût annuel $10000 \mathrm{~F}$ et de facteur de décontamination 10), à placer en série, en vue de réduire une dose collective initiale de 1 homme-sievert/an ; on se bornera à 3 filtres pour fixer les idées.

On obtient ainsi 4 options envisageables :
1) Ne rien faire ;
2) Placer 1 filtre ;
3) Placer 2 filtres en série ;
4) Placer 3 filtres en série. 


\section{Exemple (suite)}

Voici le tableau qui en résume les coûts et les performances sur le plan de la réduction de la dose collective initiale.

\begin{tabular}{|c|c|c|c|}
\hline Options & $\begin{array}{c}\text { Coût } \\
\text { Ci (kF/an) }\end{array}$ & $\begin{array}{c}\text { Dose collective } \\
\text { initiale } \\
\text { (h.Sv/an) }\end{array}$ & $\begin{array}{c}\text { Dose collective } \\
\text { finale } \\
\text { (h.Sv/an) }\end{array}$ \\
\hline 1 & 0 & 1 & 1 \\
2 & 10 & 1 & 0,1 \\
3 & 20 & 1 & 0,01 \\
4 & 30 & 1 & 0,001 \\
\hline
\end{tabular}

L'application de la théorie exige que l'on compare à alpha les rapports coûtefficacité liés au passage d'une option à la suivante, autrement dit, liés à l'installation du "dernier" filtre envisagé. C'est ce dernier filtre qui est, par définition, "l'option marginale". Son efficacité $\Delta \mathrm{S}$ varie selon qu'il s'agit du premier, du second ou du troisième filtre :

Pour le premier, l'efficacité est $1-0,1=0,9$ h.Sv/an

Pour le deuxième, l'efficacité est $0,1-0,01=0,09 \mathrm{~h} . \mathrm{Sv} / \mathrm{an}$

Pour le troisième, l'efficacité est $0,01-0,001=0,009$ h.Sv/an

Les rapports coût-efficacité $\Delta \mathrm{C} / \Delta \mathrm{S}$ qu'il convient de comparer à alpha sont, par conséquent :

Pour le premier, $\Delta C=10-0=10 \mathrm{kF} / \mathrm{an}, \Delta \mathrm{S}=0,9 \mathrm{~h} . \mathrm{Sv} / \mathrm{an}$

soit $\triangle \mathrm{C} / \triangle \mathrm{S} \simeq 11 \mathrm{kF} / \mathrm{h}$.Sv

Pour le deuxième, $\triangle \mathrm{C}=20-10=10 \mathrm{kF} / \mathrm{an}, \Delta \mathrm{S}=0,09 \mathrm{~h} . \mathrm{Sv} / \mathrm{an}$ soit $\Delta \mathrm{C} / \triangle \mathrm{S} \simeq 110 \mathrm{kF} / \mathrm{h} . \mathrm{Sv}$

Pour le troisième, $\triangle \mathrm{C}=30-20=10 \mathrm{kF} / \mathrm{an}, \Delta \mathrm{S}=0,009 \mathrm{~h} . \mathrm{Sv} / \mathrm{an}$ soit $\Delta \mathrm{C} / \Delta \mathrm{S} \simeq 1100 \mathrm{kF} / \mathrm{h}$.Sv

II faut se garder d'une interprétation erronée qui consisterait à estimer les performances et les coûts attachés aux options en se reférant cette fois-ci à la situation d'origine (aucun filtre installé) et non plus à l'option qui précède.

Autrement dit, le rapport coût-efficacité serait alors :

Pour le premier, $\triangle \mathrm{C}=10-0=10 \mathrm{kF} / \mathrm{an}, \Delta \mathrm{S}=1-0,1=0,9 \mathrm{~h} . \mathrm{Sv} / \mathrm{an}$ soit $\triangle \mathrm{C} / \triangle \mathrm{S} \simeq 11 \mathrm{kF} / \mathrm{h}$.Sv

Pour le deuxième, $\Delta \mathrm{C}=20-0=20 \mathrm{kF} / \mathrm{an}, \Delta \mathrm{S}=1-0,01=0,99 \mathrm{~h} . \mathrm{Sv} / \mathrm{an}$ soit $\triangle \mathrm{C} / \triangle \mathrm{S} \simeq 20 \mathrm{kF} / \mathrm{h}$.Sv

Pour le troisième, $\Delta \mathrm{C}=30-0=30 \mathrm{kF} / \mathrm{an}, \Delta \mathrm{S}=1-0,001=0,999 \mathrm{~h} . \mathrm{Sv} / \mathrm{an}$ soit $\triangle \mathrm{C} / \triangle \mathrm{S} \simeq 30 \mathrm{kF} / \mathrm{h}$.Sv.

Cette interprétation est, sans aucun doute, erronée si l'on se réfère à la théorie et conduirait en pratique à des conclusions fallacieuses. Ainsi, dans l'exemple traité ici, le rapport coût-efficacité de l'option 3, c'est-à-dire de l'ensemble constitué par les 3 filtres en série est de $30 \mathrm{kF} / \mathrm{h}$.Sv. Alors que le rapport lié au passage de l'option 2 à l'option 3 , seul théoriquement valable dans le contexte de l'évaluation, est de $1100 \mathrm{kF} / \mathrm{h}$.Sv, adopter la définition erronée plutôt que la définition marginale conduirait à un rapport environ 40 fois plus faible ! On conçoit aisément l'influence prépondérante de l'application correcte de la théorie sur les conclusions que l'on est susceptible d'en tirer. 


\section{II.2. L'utilisation de "alpha” selon la nature des décisions}

L'exposé des divers éléments séquentiels de l'évaluation suggère clairement qu'il s'agit d'une procédure relativement lourde si on entend la conduire jusqu'à son terme, et il convient par conséquent de s'interroger sur les limites de son champ d'application.

Le coût et la durée d'une telle étude dépendront du problème à traiter, et constitueront, bien entendu, un facteur dont il faudra tenir compte avant de décider de l'effectuer. Si le coût des options à choisir est faible, à tel point que l'étude nécessiterait un investissement supérieur à ce coût, il y a lieu de restreindre l'ambition de l'étude sans toutefois y renoncer complètement (il en va de même si les expositions mises en jeu sont très faibles).

II semble, en effet, possible d'adapter l'effort d'étude à l'ordre de grandeur économique des choix de protection. On peut distinguer, à titre illustratif, trois niveaux de décision de protection selon qu'ils mettent en jeu des coûts (ou des expositions) élevés, moyens ou faibles. Selon ces niveaux de décision, il conviendrait de traiter une partie ou l'ensemble des étapes de la procédure d'évaluation décrites plus haut.

Dans le cas des décisions relevant du "quotidien" de la radioprotection, mettant en jeu des coûts assez réduits, il paraît légitime de restreindre l'étude à l'étape d'analyse du problème et d'identification des options, l'estimation des performances des options et leur classement pouvant être effectués de façon plus ou moins qualitative. II est alors raisonnable de renoncer à effectuer les choix en référence au coût de l'homme-sievert alpha.

Pour les deux autres niveaux de décision, impliquant des coûts plus significatifs, la nécessité de se référer à une valeur de alpha sera d'autant plus grande que les coûts des options à choisir sont élevés. En particulier, l'application de la procédure d'évaluation menée jusqu'à son étape ultime sera souvent indiquée dans le cas de la conception des installations. Les coûts de protection peuvent alors représenter une part relativement importante des coûts d'investissement mis en jeu.

En résumé, on peut proposer le tableau I fournissant une échelle qualitative mesurant l'importance relative des différentes étapes de la procédure d'évaluation, ceci en fonction du type de décision de radioprotection.

TABLEAU I

Importance relative des différentes étapes de la procédure d'évaluation selon l'ordre de grandeur économique des choix de protection

\begin{tabular}{|c|c|c|c|}
\hline Les étapes Coûts de protection & Elevé & Moyen & Faible \\
\hline $\begin{array}{l}\text { Analyse du problème à traiter et identifi- } \\
\text { cation des options }\end{array}$ & +++ & +++ & +++ \\
\hline Estimation des coûts et des performances & +++ & ++ & $(+)$ \\
\hline Classement des options & +++ & ++ & $(+)$ \\
\hline $\begin{array}{l}\text { Recours à alpha pour le choix de la "meilleure" } \\
\text { option }\end{array}$ & +++ & + & \\
\hline
\end{tabular}




\section{CONCLUSION}

La nécessité de rationaliser les choix en matière de protection radiologique est liée au souci d'allouer au mieux les dépenses de protection et de rendre plus explicites les critères de choix par le biais de la quantification. Cette nécessité a notamment été récemment rappelée lors du second séminaire scientifique européen sur l'optimisation de la protection radiologique [4]. Si l'examen attentif des diverses options de protection envisageables et de la définition des critères devant être intégrés dans la procédure de choix sont deux étapes indispensables, le recours à la dimension économique (coût de protection) n'est pertinent que si les dépenses sont significatives.

II convient, cependant, de rappeler que la procédure décrite ci-dessus est un outil d'aide à la décision et ne peut en aucun cas se substituer au jugement du décideur auquel incombe en définitive la responsabilité du choix. Elle permet de recenser l'ensemble des paramètres devant intervenir pour atteindre l'objectif de la protection radiologique et de procéder à une estimation prédictive des avantages et inconvénients de chacune des solutions envisagées.

\section{RÉFÉRENCES}

[1] M.J. CLARK, A.B. FLEISHMAN, G.A.M. WEBB. Optimisation of the radiological protection of the public. NRPB-R120, 1981.

[2] Commission internationale de protection radiologique (CIPR). Recommandations (Publication CIPR n²6). Oxford: Pergamon press, 1977.

[3] Commission internationale de protection radiologique (CIPR). Cost-benefit analysis in the optimisation of radiation protection (ICPR Publication 37). Oxford: Pergamon press, 1982.

[4] Communautés européennes (Commission). Radiation protection optimization "As low as reasonably achievable", 2. European scientific seminar, Luxembourg, 8-9 November 1983 (JOLIVET A., SINNAEVE J., Eds). Luxembourg : Communautés européennes, 1984 (EUR-9173).

[5] GRANDE-BRETAGNE. National radiological protection board (NRPB). Cost-benefit analysis in the optimisation of protection of radiation workers, a consultative document, Chilton : NRPB Nov. 1982.

[6] P. JAXEL, A. OUDIZ, J. LOMBARD. Recherche des valeurs implicites du détriment sanitaire dans le public - un exemple dans l'industrie chimique. Rapport CEPN n०62, 1983.

[7] C. LE GALES, A. OUDIZ. Contribution méthodologique à la détermination de valeurs limites d'exposition professionnelle à l'amiante. Rev. Fr. Epidémiol. Santé publ., 1984, 32, 113-121.

[8] J. LOCHARD, C. MACCIA, P. PAGES. Cost-effectiveness considerations in reducing occupational radiation exposure in nuclear power plants. Nucl. Safety, 1983, 24 (6) $821-828$

[9] J. LOMBARD. La prise en compte de la distribution des doses individuelles dans l'optimisation de la protection radiologique. Rapport CEPN nº65, 1983. 
[10] J. LOMBARD. La prise en compte du long terme dans l'évaluation du détriment radiologique et de son coût. Rapport CEPN n 73, 1984.

[11] J. LOMBARD, A. OUDIZ, G. UZZAN. La prise en compte du risque travailleurs dans la mise en œuvre du principe d'optimisation des doses au public - Radioprotection, 1982, 17 (3) 155-162.

[12] A. OUDIZ, J. LOMBARD, P. ZETTWOOG. Un exemple d'optimisation de la protection radiologique des mineurs d'uranium. Radioprotection, 1984, 19 (1) 23-35.

[13] A. OUDIZ, J. LOMBARD, F. FAGNANI. A multi-attribute approach to the rationalisation of radiological protection. Health Phys., 1981,40 (6) 783-799. 